Article

\title{
Some New $q$-Integral Inequalities Using Generalized Quantum Montgomery Identity via Preinvex Functions
}

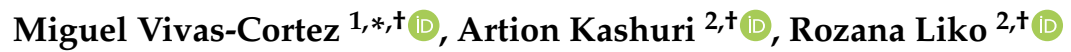 \\ and Jorge E. Hernández Hernández ${ }^{3,+} \mathbb{D}$ \\ 1 Escuela de Ciencias Físicas y Matemáticas, Facultad de Ciencias Exactas y Naturales, Pontificia Universidad \\ Católica del Ecuador, Av. 12 de Octubre 1076. Apartado, Quito 17-01-2184, Ecuador \\ 2 Department of Mathematics, Faculty of Technical Science, University Ismail Qemali, L. Pavaresia, Vlora \\ 1001, Vlore, Albania; artionkashuri@gmail.com (A.K.); rozanaliko86@gmail.com (R.L.) \\ 3 Departamento de Técnicas Cuantitativas, Decanato de Ciencias Económicas y Empresariales, Universidad \\ Centroccidental Lisandro Alvarado, Av. 20. esq. Av. Moran, Edf. Los Militares, Piso 2, Ofc.2, \\ Barquisimeto 3001, Venezuela; jorgehernandez@ucla.edu.ve \\ * Correspondence: mjvivas@puce.edu.ec \\ + These authors contributed equally to this work.
}

Received: 31 January 2020; Accepted: 26 February 2020; Published: 4 April 2020

check for updates

\begin{abstract}
In this work the authors establish a new generalized version of Montgomery's identity in the setting of quantum calculus. From this result, some new estimates of Ostrowski type inequalities are given using preinvex functions. Given the generality of preinvex functions, particular $q$-integral inequalities are established with appropriate choice of the parametric bifunction. Some new special cases from the main results are obtained and some known results are recaptured as well. At the end, a briefly conclusion is given.
\end{abstract}

Keywords: Quantum Montgomery identity; $\phi$-convex functions; integral inequalities

MSC: 26D10; 52A01; 26D07

\section{Introduction}

Quantum calculus, or $q$-calculus, has had an important development in recent decades, both in pure mathematics and its applicability, for example in Physics [1]. The convexity of a function has played an important role as a tool in the development of inequalities. Some fields of Mathematics have used this property: harmonic analysis, interpolation theory, and control theory, as can be seen in the works of C.P. Niculescu [2], C. Bennett and R. Sharpley [3], S. Mititelu and S. Trenţă [4], S. Trenţă [5,6].

Furthermore, it is important to note that in recent decades, the evolution of the concept of convexity has been extended and its evolution has been subject of many studies as is shown in the works of Ben-Israel A. and Mond B. [7], Hernández Hernández, J. E. [8,9], Niculescu C.P. [2], Mitrinović D.S. et al. [10], Noor M. et.al. [11,12], Sarikaya M.Z. et. al. [13], Vivas-Cortez M.J. et al. [14], Weir, T.; Mond, B. [15] and others.

Recently, Tariboon et al. in [16], defined q-derivative and q-integral as follows:

Definition 1. Let $\mathrm{Y}:\left[a_{1}, a_{2}\right] \rightarrow \mathbb{R}$ be a continuous function and let $x \in\left[a_{1}, a_{2}\right]$ and $0<\mathrm{q}<1$ be a constant. Then the q-derivative on $\left[a_{1}, a_{2}\right]$ of function $\mathrm{Y}$ at $x$ is defined as

$$
a_{1} D_{\mathrm{q}} \mathrm{Y}(x)=\frac{\mathrm{Y}(x)-\mathrm{Y}\left(q x+(1-\mathrm{q}) a_{1}\right)}{(1-\mathrm{q})\left(x-a_{1}\right)}, x \neq a_{1}
$$


We say that $\mathrm{Y}$ is q-differentiable on $\left[a_{1}, a_{2}\right]$ provided ${ }_{a_{1}} D_{\mathrm{q}} \mathrm{Y}(x)$ exists for all $x \in\left[a_{1}, a_{2}\right]$.

Definition 2. Let $\mathrm{Y}:\left[a_{1}, a_{2}\right] \subset \mathbb{R} \rightarrow \mathbb{R}$ be a continuous function. Then $\mathrm{q}$-integral on $\left[a_{1}, a_{2}\right]$ is defined as

$$
\int_{a_{1}}^{x} \mathrm{Y}(v)_{a_{1}} d_{\mathrm{q}} v=(1-\mathrm{q})\left(x-a_{1}\right) \sum_{n=0}^{\infty} \mathrm{q}^{n} \mathrm{Y}\left(\mathrm{q}^{n} x+\left(1-\mathrm{q}^{n}\right) a_{1}\right),
$$

for $x \in\left[a_{1}, a_{2}\right]$.

Some properties of interest regarding these definitions are the following.

Theorem 1. ([17]) Let $f: J \rightarrow \mathbb{R}$ be a $q$-differentiable functions. Then we have

1. The sum $(f+g)$ is $q$-differentiable on J with

$$
{ }_{a} D_{q}(f(t)+g(t))={ }_{a} D_{q} f(t)+{ }_{a} D_{q} g(t)
$$

2. For any constant $\alpha \in \mathbb{R}$, the function ( $\alpha f$ is $q$-differentiable and

$$
{ }_{a} D_{q}(\alpha f(t))=\alpha_{a} D_{q} f(t)
$$

3. The function $(f g)$ is $q$-differentiable with

$$
\begin{aligned}
{ }_{a} D_{q}(f g)(t) & =f(t)_{a} D_{q} g(t)+g(q t+(1-q) a)_{a} D_{q} f(t) \\
& =g(t)_{a} D_{q} f(t)+f(q t+(1-q) a)_{a} D_{q} g(t)
\end{aligned}
$$

Lemma 1. [17]. Let $\alpha \in \mathbb{R}$, then we have

$$
{ }_{a} D_{q}(x-a)^{\alpha}=\left(\frac{1-q^{\alpha}}{1-q}\right)(x-a)^{\alpha-1}
$$

Theorem 2. [17] Let $f: J \rightarrow \mathbb{R}$ be a continuous function. Then we have

1. ${ }_{a} D_{q} \int_{a}^{x} f(t)_{a} d_{q} t=f(x)$

2. $\int_{c}^{x}{ }_{a} D_{q} f(t) d_{a} d_{q} t=f(x)-f(c)$ for $c \in(a, x)$

Theorem 3. [17] Let $f, g: J \rightarrow \mathbb{R}$ be a continuous functions and $\alpha \in \mathbb{R}$. Then, for $x \in J$ we have

1. $\int_{a_{x}}^{x}(f(t)+g(t))_{a} d_{q} t=\int_{a}^{x} f(t)_{a} d_{q} t+\int_{a}^{x} g(t)_{a} d_{q} t$

2. $\int_{a}^{x}(\alpha f(t))_{a} d_{q} t=\alpha \int_{a}^{x} f(t)_{a} d_{q} t$

3. $\int_{c}^{x} f(t)_{a} D_{q} g(t)_{a} d_{q} t=\left.f(t) g(t)\right|_{c} ^{x}-\int_{c}^{x} g(q t+(1-q) a)_{a} D_{q} f(t)_{a} d_{q} t$

For more details on q-calculus and certain q-analogues of classical inequalities, see [16,18-29].

The following famous identity in [10], is called Montgomery identity:

$$
f(x)=\frac{1}{a_{2}-a_{1}} \int_{a_{1}}^{a_{2}} f(v) d v+\frac{1}{a_{2}-a_{1}} \int_{a_{1}}^{x}\left(v-a_{1}\right) f^{\prime}(v) d v+\frac{1}{a_{2}-a_{1}} \int_{x}^{a_{2}}\left(v-a_{2}\right) f^{\prime}(v) d v,
$$

where $f(x)$ is continuous function on $\left[a_{1}, a_{2}\right]$ with a continuous first derivative in $\left(a_{1}, a_{2}\right)$. By changing the variable, the Montgomery identity (3) could be expressed as follows:

$$
f(x)-\frac{1}{a_{2}-a_{1}} \int_{a_{1}}^{a_{2}} f(v) d v=\left(a_{2}-a_{1}\right) \int_{0}^{1} K(v) f^{\prime}\left((1-v) a_{1}+v a_{2}\right) d v,
$$


where

$$
K(v)= \begin{cases}v & , \quad v \in\left[0, \frac{x-a_{1}}{a_{2}-a_{1}}\right], \\ v-1, & v \in\left[\frac{x-a_{1}}{a_{2}-a_{1}}, 1\right] .\end{cases}
$$

This identity has been used in various works to establish bounds for quadrature rules via specialized algorithms [30].

We recall now some basic definitions for our study as follows:

Let $\mathbf{E} \subset \mathbb{R}^{n}$ be a non-empty set, $\mathrm{Y}: \mathbf{E} \rightarrow \mathbb{R}$ be a continuous functions and $\theta: \mathbf{E} \times \mathbf{E} \rightarrow \mathbb{R}^{n}$ be a continuous bifunction.

Definition 3. [7] A set $\mathbf{E} \subset \mathbb{R}^{n}$ is said to be invex with respect to bifunction $\theta(.,$.$) , if$

$$
a_{1}+v \theta\left(a_{2}, a_{1}\right) \in \mathbf{E}, \quad \forall a_{1}, a_{2} \in \mathbf{E}, v \in[0,1] .
$$

Definition 4. [15] A function $\mathrm{Y}: \mathrm{E} \rightarrow \mathbb{R}$ is said to be preinvex with respect to bifunction $\theta(.,$.$) , if$

$$
\mathrm{Y}\left(a_{1}+v \theta\left(a_{2}, a_{1}\right)\right) \leq(1-v) \mathrm{Y}\left(a_{1}\right)+v \mathrm{Y}\left(a_{2}\right), \quad \forall a_{1}, a_{2} \in \mathbf{E}, v \in[0,1] .
$$

Some properties of this class of functions can be found in [31].

Motivated by the above literatures, the main objective of this article is to obtain a generalization of the Montgomery identity given in (4) using the concepts of q-calculus. From this identity, several new and known q-analogues of integral inequalities involving preinvex functions will be obtain. We also discuss some new special cases of the main results. At the end, a briefly conclusion is provided as well.

\section{Main Results}

In this section, before we derive our main results, for brevity we define the following notations:

$$
P=\left[a_{1}, a_{1}+\theta\left(a_{2}, a_{1}\right)\right], \quad \wp_{\theta}(x)=\frac{x-a_{1}}{\theta\left(a_{2}, a_{1}\right)}, \text { where } \theta\left(a_{2}, a_{1}\right)>0 .
$$

Lemma 2. (Generalized quantum Montgomery identity) If $\mathrm{Y}: P \rightarrow \mathbb{R}$ is a q-differentiable function such that ${ }_{e} D_{\mathrm{q}} \mathrm{Y}$ is quantum integrable on $P^{\circ}$ (the interior of $P$ ), then the following identity holds:

$$
\mathrm{Y}(x)-\frac{1}{\theta\left(a_{2}, a_{1}\right)} \int_{a_{1}}^{a_{1}+\theta\left(a_{2}, a_{1}\right)} \mathrm{Y}(v)_{a_{1}} d_{\mathrm{q}} v=\theta\left(a_{2}, a_{1}\right) \int_{0}^{1} T_{\mathrm{q}}(v)_{a_{1}} D_{\mathrm{q}} \mathrm{Y}\left(a_{1}+v \theta\left(a_{2}, a_{1}\right)\right)_{0} d_{\mathrm{q}} v,
$$

where

$$
T_{\mathrm{q}}(v)=\left\{\begin{array}{r}
\mathrm{q} v, \quad \text { if } \quad v \in\left[0, \wp_{\theta}(x)\right] \\
\mathrm{q} v-1, \quad \text { if } \quad v \in\left(\wp_{\theta}(x), 1\right]
\end{array}\right.
$$


Proof. By using Definitions 1 and 2, we have

$$
\begin{aligned}
& \theta\left(a_{2}, a_{1}\right) \int_{0}^{1} T_{\mathrm{q}}(v) a_{1} D_{\mathrm{q}} \mathrm{Y}\left(a_{1}+v \theta\left(a_{2}, a_{1}\right)\right){ }_{0} d_{\mathrm{q}} v \\
& =\theta\left(a_{2}, a_{1}\right)\left[\int_{0}^{\wp_{\theta}(x)} \mathrm{q} v_{a_{1}} D_{\mathrm{q}} \mathrm{Y}\left(a_{1}+v \theta\left(a_{2}, a_{1}\right)\right)_{0} d_{\mathrm{q}} v+\int_{\wp_{\theta}(x)}^{1}(\mathrm{q} v-1) a_{1} D_{\mathrm{q}} \mathrm{Y}\left(a_{1}+v \theta\left(a_{2}, a_{1}\right)\right){ }_{0} d_{\mathrm{q}} v\right] \\
& =\theta\left(a_{2}, a_{1}\right)\left[\int_{0}^{\wp_{\theta}(x)} \mathrm{q} v_{a_{1}} D_{\mathrm{q}} \mathrm{Y}\left(a_{1}+v \theta\left(a_{2}, a_{1}\right)\right){ }_{0} d_{\mathrm{q}} v+\int_{0}^{1}(\mathrm{q} v-1) a_{1} D_{\mathrm{q}} \mathrm{Y}\left(a_{1}+v \theta\left(a_{2}, a_{1}\right)\right)_{0} d_{\mathrm{q}} v\right. \\
& \left.-\int_{0}^{\wp_{\theta}(x)}(\mathrm{q} v-1) a_{1} D_{\mathrm{q}} \mathrm{Y}\left(a_{1}+v \theta\left(a_{2}, a_{1}\right)\right){ }_{0} d_{\mathrm{q}} v\right] \\
& =\theta\left(a_{2}, a_{1}\right)\left[\int_{0}^{1}(\mathrm{q} v-1) a_{1} D_{\mathrm{q}} \mathrm{Y}\left(a_{1}+v \theta\left(a_{2}, a_{1}\right)\right){ }_{0} d_{\mathrm{q}} v+\int_{0}^{\wp_{\theta}(x)} a_{1} D_{\mathrm{q}} \mathrm{Y}\left(a_{1}+v \theta\left(a_{2}, a_{1}\right)\right){ }_{0} d_{\mathrm{q}} v\right] \\
& =\theta\left(a_{2}, a_{1}\right)\left[\int_{0}^{1} \mathrm{q} v_{a_{1}} D_{\mathrm{q}} \mathrm{Y}\left(a_{1}+v \theta\left(a_{2}, a_{1}\right)\right){ }_{0} d_{\mathrm{q}} v-\int_{0}^{1} a_{1} D_{\mathrm{q}} \mathrm{Y}\left(a_{1}+v \theta\left(a_{2}, a_{1}\right)\right){ }_{0} d_{\mathrm{q}} v\right. \\
& \left.+\int_{0}^{\wp_{\theta}(x)} a_{1} D_{\mathrm{q}} \mathrm{Y}\left(a_{1}+v \theta\left(a_{2}, a_{1}\right)\right)_{0} d_{\mathrm{q}} v\right] \\
& =\frac{1}{1-\mathrm{q}}\left[\mathrm{q}\left[\int_{0}^{1} \mathrm{Y}\left(a_{1}+v \theta\left(a_{2}, a_{1}\right)\right){ }_{0} d_{\mathrm{q}} v-\int_{0}^{1} \mathrm{Y}\left(a_{1}+\mathrm{q} v \theta\left(a_{2}, a_{1}\right)\right){ }_{0} d_{\mathrm{q}} v\right]\right. \\
& -\left[\int_{0}^{1} \frac{\mathrm{Y}\left(a_{1}+v \theta\left(a_{2}, a_{1}\right)\right)}{v} d_{\mathrm{q}} v-\int_{0}^{1} \frac{\mathrm{Y}\left(a_{1}+\mathrm{q} v \theta\left(a_{2}, a_{1}\right)\right)}{v} d_{\mathrm{q}} v\right] \\
& \left.+\left[\int_{0}^{\wp_{\theta}(x)} \frac{\mathrm{Y}\left(a_{1}+v \theta\left(a_{2}, a_{1}\right)\right)}{v} d_{\mathrm{q}} v-\int_{0}^{\wp_{\theta}(x)} \frac{\mathrm{Y}\left(a_{1}+\mathrm{q} v \theta\left(a_{2}, a_{1}\right)\right)}{v} d_{\mathrm{q}} v\right]\right]
\end{aligned}
$$




$$
\begin{aligned}
& =\frac{1}{1-\mathrm{q}}\left[\mathrm{q}(1-\mathrm{q})\left(\sum_{n=0}^{\infty} \mathrm{q}^{n} \mathrm{Y}\left(a_{1}+\mathrm{q}^{n} \theta\left(a_{2}, a_{1}\right)\right)-\sum_{n=0}^{\infty} \mathrm{q}^{n} \mathrm{Y}\left(a_{1}+\mathrm{q}^{n+1} \theta\left(a_{2}, a_{1}\right)\right)\right)\right. \\
& -(1-\mathrm{q})\left(\sum_{n=0}^{\infty} \mathrm{q}^{n} \frac{\mathrm{Y}\left(a_{1}+\mathrm{q}^{n} \theta\left(a_{2}, a_{1}\right)\right)}{\mathrm{q}^{n}}-\sum_{n=0}^{\infty} \mathrm{q}^{n} \frac{\mathrm{Y}\left(a_{1}+\mathrm{q}^{n+1} \theta\left(a_{2}, a_{1}\right)\right)}{\mathrm{q}^{n}}\right) \\
& \left.+(1-\mathrm{q}) \wp_{\theta}(x)\left(\sum_{n=0}^{\infty} \mathrm{q}^{n} \frac{\mathrm{Y}\left(a_{1}+\mathrm{q}^{n} \wp_{\theta}(x) \theta\left(a_{2}, a_{1}\right)\right)}{\mathrm{q}^{n} \wp_{\theta}(x)}-\sum_{n=0}^{\infty} \mathrm{q}^{n} \frac{\mathrm{Y}\left(a_{1}+\mathrm{q}^{n+1} \wp_{\theta}(x) \theta\left(a_{2}, a_{1}\right)\right)}{\mathrm{q}^{n} \wp_{\theta}(x)}\right)\right] \\
& =\mathrm{q}\left(\sum_{n=0}^{\infty} \mathrm{q}^{n} \mathrm{Y}\left(a_{1}+\mathrm{q}^{n} \theta\left(a_{2}, a_{1}\right)\right)-\sum_{n=0}^{\infty} \mathrm{q}^{n} \mathrm{Y}\left(a_{1}+\mathrm{q}^{n+1} \theta\left(a_{2}, a_{1}\right)\right)\right) \\
& -\left(\sum_{n=0}^{\infty} \mathrm{Y}\left(a_{1}+\mathrm{q}^{n} \theta\left(a_{2}, a_{1}\right)\right)-\sum_{n=0}^{\infty} \mathrm{Y}\left(a_{1}+\mathrm{q}^{n+1} \theta\left(a_{2}, a_{1}\right)\right)\right) \\
& +\left(\sum_{n=0}^{\infty} \mathrm{Y}\left(a_{1}+\mathrm{q}^{n} \wp_{\theta}(x) \theta\left(a_{2}, a_{1}\right)\right)-\sum_{n=0}^{\infty} \mathrm{Y}\left(a_{1}+\mathrm{q}^{n+1} \wp_{\theta}(x) \theta\left(a_{2}, a_{1}\right)\right)\right) \\
& =\mathrm{q}\left(\sum_{n=0}^{\infty} \mathrm{q}^{n} \mathrm{Y}\left(a_{1}+\mathrm{q}^{n} \theta\left(a_{2}, a_{1}\right)\right)-\frac{1}{\mathrm{q}} \sum_{n=1}^{\infty} \mathrm{q}^{n} \mathrm{Y}\left(a_{1}+\mathrm{q}^{n} \theta\left(a_{2}, a_{1}\right)\right)\right) \\
& -\left(\sum_{n=0}^{\infty} \mathrm{Y}\left(a_{1}+\mathrm{q}^{n} \theta\left(a_{2}, a_{1}\right)\right)-\sum_{n=1}^{\infty} \mathrm{Y}\left(a_{1}+\mathrm{q}^{n} \theta\left(a_{2}, a_{1}\right)\right)\right) \\
& +\left(\sum_{n=0}^{\infty} \mathrm{Y}\left(a_{1}+\mathrm{q}^{n} \wp_{\theta}(x) \theta\left(a_{2}, a_{1}\right)\right)-\sum_{n=1}^{\infty} \mathrm{Y}\left(a_{1}+\mathrm{q}^{n} \wp_{\theta}(x) \theta\left(a_{2}, a_{1}\right)\right)\right) \\
& =\mathrm{q}\left[\left(1-\frac{1}{\mathrm{q}}\right) \sum_{n=0}^{\infty} \mathrm{q}^{n} \mathrm{Y}\left(a_{1}+\mathrm{q}^{n} \theta\left(a_{2}, a_{1}\right)\right)+\frac{\mathrm{Y}\left(a_{1}+\theta\left(a_{2}, a_{1}\right)\right)}{\mathrm{q}}\right] \\
& -\mathrm{Y}\left(a_{1}+\theta\left(a_{2}, a_{1}\right)\right)+\mathrm{Y}\left(a_{1}+\wp_{\theta}(x) \theta\left(a_{2}, a_{1}\right)\right) \\
& =\mathrm{Y}(x)-(1-\mathrm{q}) \sum_{n=0}^{\infty} \mathrm{q}^{n} \mathrm{Y}\left(a_{1}+\mathrm{q}^{n} \theta\left(a_{2}, a_{1}\right)\right) \\
& =\mathrm{Y}(x)-\frac{1}{\theta\left(a_{2}, a_{1}\right)} \int_{a_{1}}^{a_{1}+\theta\left(a_{2}, a_{1}\right)} \mathrm{Y}(v)_{a_{1}} d_{\mathrm{q}} v .
\end{aligned}
$$

The proof is complete.

Remark 1. Taking $\mathrm{q} \rightarrow 1^{-}$in Lemma 2, we have

$$
\begin{aligned}
& \mathrm{Y}(x)-\frac{1}{\theta\left(a_{2}, a_{1}\right)} \int_{a_{1}}^{a_{1}+\theta\left(a_{2}, a_{1}\right)} \mathrm{Y}(v) d v \\
& =\theta\left(a_{2}, a_{1}\right)\left[\int_{0}^{\wp_{\theta}(x)} v \mathrm{Y}^{\prime}\left(a_{1}+v \theta\left(a_{2}, a_{1}\right)\right) d v+\int_{\wp_{\theta}(x)}^{1}(v-1) \mathrm{Y}^{\prime}\left(a_{1}+v \theta\left(a_{2}, a_{1}\right)\right) d v\right] .
\end{aligned}
$$

Remark 2. Taking $\theta\left(a_{2}, a_{1}\right)=a_{2}-a_{1}$ in Lemma 2, we get ([20], Lemma 3).

Remark 3. Taking $\mathrm{q} \rightarrow 1^{-}$and $\theta\left(a_{2}, a_{1}\right)=a_{2}-a_{1}$ in Lemma 2, we obtain Montgomery identity given in (4). 
Remark 4. Taking $x=\frac{2 a_{1}+\theta\left(a_{2}, a_{1}\right)}{2}$ in Remark 1, we get ([11], Lemma 3.10).

$$
\begin{aligned}
& Y\left(\frac{2 a_{1}+\theta\left(a_{2}, a_{1}\right)}{2}\right)-\frac{1}{\theta\left(a_{2}, a_{1}\right)} \int_{a_{1}}^{a_{1}+v \theta\left(a_{2}, a_{1}\right)} Y(v) d v \\
& =\theta\left(a_{2}, a_{1}\right)\left[\int_{0}^{\frac{1}{2}} v Y^{\prime}\left(a_{1}+v \theta\left(a_{2}, a_{1}\right)\right) d v+\int_{\frac{1}{2}}^{1}(v-1) Y^{\prime}\left(a_{1}+v \theta\left(a_{2}, a_{1}\right)\right) d v\right] .
\end{aligned}
$$

Remark 5. Taking $x=\frac{q a_{1}+a_{2}}{1+q}$ and $\theta\left(a_{2}, a_{1}\right)=a_{2}-a_{1}$ in Lemma 2, we obtain equality (4.1) of [18].

$$
\begin{aligned}
\mathrm{Y}\left(\frac{q a_{1}+a_{2}}{1+\mathrm{q}}\right)-\frac{1}{a_{2}-a_{1}} \int_{a_{1}}^{a_{2}} \mathrm{Y}(v)_{a_{1}} d_{\mathrm{q}} v \\
=\left(a_{2}-a_{1}\right)\left[\int_{0}^{\frac{1}{1+\mathrm{q}}} \mathrm{q} v_{a_{1}} D_{\mathrm{q}} \mathrm{Y}\left((1-v) a_{1}+v a_{2}\right)_{0} d_{\mathrm{q}} v\right. \\
\left.+\int_{\frac{1}{1+\mathrm{q}}}^{1}(\mathrm{q} v-1)_{a_{1}} D_{\mathrm{q}} \mathrm{Y}\left((1-v) a_{1}+v a_{2}\right)_{0} d_{\mathrm{q}} v\right] .
\end{aligned}
$$

Remark 6. Taking $x=\frac{a_{1}+\mathrm{q}\left(a_{1}+\theta\left(a_{2}, a_{1}\right)\right)}{1+\mathrm{q}}$ in Lemma 2, we have

$$
\begin{aligned}
\mathrm{Y}\left(\frac{a_{1}+\mathrm{q}\left(a_{1}+\theta\left(a_{2}, a_{1}\right)\right)}{1+\mathrm{q}}\right)-\frac{1}{\theta\left(a_{2}, a_{1}\right)} & \int_{a_{1}}^{a_{1}+\theta\left(a_{2}, a_{1}\right)} \mathrm{Y}(v) a_{1} d_{\mathrm{q}} v \\
=\theta\left(a_{2}, a_{1}\right) & {\left[\int_{0}^{\frac{\mathrm{q}}{1+\mathrm{q}}} \mathrm{q} v_{a_{1}} D_{\mathrm{q}} \mathrm{Y}\left(a_{1}+v \theta\left(a_{2}, a_{1}\right)\right)_{0} d_{\mathrm{q}} v\right.} \\
& \left.+\int_{\frac{\mathrm{q}}{1+\mathrm{q}}}^{1}(\mathrm{q} v-1)_{a_{1}} D_{\mathrm{q}} \mathrm{Y}\left(a_{1}+v \theta\left(a_{2}, a_{1}\right)\right){ }_{0} d_{\mathrm{q}} v\right] .
\end{aligned}
$$

Now using Lemma 2, we are in position to derive our main results for the class of preinvex functions.

Theorem 4. Let $\mathrm{Y}: P \rightarrow \mathbb{R}$ be a function such that $a_{1} D_{\mathrm{q}} \mathrm{Y}$ is q-integrable on $P^{\circ}$ (the interior of $P$ ). If $\left|a_{1} D_{\mathrm{q}} \mathrm{Y}\right|^{r}$ is preinvex function on $P$, then for $r>1$ and $p^{-1}+r^{-1}=1$, the following inequality holds:

$$
\begin{aligned}
& \left|\mathrm{Y}(x)-\frac{1}{\theta\left(a_{2}, a_{1}\right)} \int_{a_{1}}^{a_{1}+\theta\left(a_{2}, a_{1}\right)} \mathrm{Y}(v)_{a_{1}} d_{\mathrm{q}} v\right| \\
& \leq \mathrm{q} \theta\left(a_{2}, a_{1}\right)\left[\left(L_{1}\left(\mathrm{q}, a_{1}, a_{2}, x\right)\right)^{\frac{1}{p}}\left(\left|a_{1} D_{\mathrm{q}} \mathrm{Y}\left(a_{1}\right)\right|^{r} L_{2}\left(\mathrm{q}, a_{1}, a_{2}, x\right)+\left.\left.\right|_{a_{1}} D_{\mathrm{q}} \mathrm{Y}\left(a_{2}\right)\right|^{r} L_{3}\left(\mathrm{q}, a_{1}, a_{2}, x\right)\right)^{\frac{1}{r}}\right. \\
& \left.+\left(L_{4}\left(\mathrm{q}, a_{1}, a_{2}, x\right)\right)^{\frac{1}{p}}\left(\left|a_{1} D_{\mathrm{q}} \mathrm{Y}\left(a_{1}\right)\right|^{r} L_{5}\left(\mathrm{q}, a_{1}, a_{2}, x\right)+\left.\left.\right|_{a_{1}} D_{\mathrm{q}} \mathrm{Y}\left(a_{2}\right)\right|^{r} L_{6}\left(\mathrm{q}, a_{1}, a_{2}, x\right)\right)^{\frac{1}{r}}\right],
\end{aligned}
$$


where

$$
\begin{aligned}
& L_{1}\left(\mathrm{q}, a_{1}, a_{2}, x\right)=\left[\wp_{\theta}(x)\right]^{p+1} \frac{1-\mathrm{q}}{1-\mathrm{q}^{p+1}}, \\
& L_{2}\left(\mathrm{q}, a_{1}, a_{2}, x\right)=\wp_{\theta}(x)-\frac{1}{1+\mathrm{q}}\left[\wp_{\theta}(x)\right]^{2}, \\
& L_{3}\left(\mathrm{q}, a_{1}, a_{2}, x\right)=\frac{1}{1+\mathrm{q}}\left[\wp_{\theta}(x)\right]^{2}, \\
& L_{4}\left(\mathrm{q}, a_{1}, a_{2}, x\right)=(1-\mathrm{q})\left[\sum_{n=0}^{\infty} \mathrm{q}^{n}\left(\mathrm{q}^{n}-\frac{1}{\mathrm{q}}\right)^{p}-\wp_{\theta}(x) \sum_{n=0}^{\infty} \mathrm{q}^{n}\left(\mathrm{q}^{n} \wp_{\theta}(x)-\frac{1}{\mathrm{q}}\right)^{p}\right], \\
& L_{5}\left(\mathrm{q}, a_{1}, a_{2}, x\right)=\frac{\mathrm{q}}{1+\mathrm{q}}-\wp_{\theta}(x)+\frac{1}{1+\mathrm{q}}\left[\wp_{\theta}(x)\right]^{2}, \\
& L_{6}\left(\mathrm{q}, a_{1}, a_{2}, x\right)=\frac{1}{1+\mathrm{q}}\left(1-\left[\wp_{\theta}(x)\right]^{2}\right) .
\end{aligned}
$$

Proof. Using Lemma 2, preinvexity of $\left|{ }_{a_{1}} D_{\mathrm{q}} Y\right|^{r}$ and Hölder's inequality, we get

$$
\begin{aligned}
& \left|Y(x)-\frac{1}{\theta\left(a_{2}, a_{1}\right)} \int_{a_{1}}^{a_{1}+\theta\left(a_{2}, a_{1}\right)} Y(v) a_{1} d_{\mathrm{q}} v\right| \\
& \leq \theta\left(a_{2}, a_{1}\right)\left[\left.\left.\int_{0}^{\wp_{\theta}(x)} \mathrm{q} v\right|_{a_{1}} D_{\mathrm{q}} \mathrm{Y}\left(a_{1}+v \theta\left(a_{2}, a_{1}\right)\right)\right|_{0} d_{\mathrm{q}} v\right. \\
& \left.+\left.\left.\int_{\wp_{\theta}(x)}^{1}(\mathrm{q} v-1)\right|_{a_{1}} D_{\mathrm{q}} \mathrm{Y}\left(a_{1}+v \theta\left(a_{2}, a_{1}\right)\right)\right|_{0} d_{\mathrm{q}} v\right] \\
& \leq \theta\left(a_{2}, a_{1}\right)\left[\left(\int_{0}^{\wp_{\theta}(x)}(\mathrm{q} v)^{p}{ }_{0} d_{\mathrm{q}} v\right)^{\frac{1}{p}}\left(\int_{0}^{\wp_{\theta}(x)}\left|a_{1} D_{\mathrm{q}} \mathrm{Y}\left(a_{1}+v \theta\left(a_{2}, a_{1}\right)\right)\right|^{r}{ }_{0} d_{\mathrm{q}} v\right)^{\frac{1}{r}}\right. \\
& \left.+\left(\int_{\sigma_{\theta}(x)}^{1}(\mathrm{q} v-1)^{p}{ }_{0} d_{\mathrm{q}} v\right)^{\frac{1}{p}}\left(\int_{\sigma_{\theta}(x)}^{1}\left|a_{1} D_{\mathrm{q}} \mathrm{Y}\left(a_{1}+v \theta\left(a_{2}, a_{1}\right)\right)\right|^{r}{ }_{0} d_{\mathrm{q}} v\right)^{\frac{1}{r}}\right] \\
& \leq \mathrm{q} \theta\left(a_{2}, a_{1}\right)\left[\left(\int_{0}^{\wp_{\theta}(x)} v^{p}{ }_{0} d_{\mathrm{q}} v\right)^{\frac{1}{p}}\left(\left|a_{1} D_{\mathrm{q}} \mathrm{Y}\left(a_{1}\right)\right|^{r} \int_{0}^{\wp_{\theta}(x)}(1-v){ }_{0} d_{\mathrm{q}} v+\left|a_{1} D_{\mathrm{q}} \mathrm{Y}\left(a_{2}\right)\right|^{r} \int_{0}^{\wp_{\theta}(x)} v_{0} d_{\mathrm{q}} v\right)^{\frac{1}{r}}\right. \\
& \left.+\left(\int_{\wp_{\theta}(x)}^{1}\left(v-\frac{1}{\mathrm{q}}\right)^{p}{ }_{0} d_{\mathrm{q}} v\right)^{\frac{1}{p}}\left(\left|a_{1} D_{\mathrm{q}} \mathrm{Y}\left(a_{1}\right)\right|^{r} \int_{\wp_{\theta}(x)}^{1}(1-v)_{0} d_{\mathrm{q}} v+\left|a_{1} D_{\mathrm{q}} \mathrm{Y}\left(a_{2}\right)\right|^{r} \int_{\wp_{\theta}(x)}^{1} v_{0} d_{\mathrm{q}} v\right)^{\frac{1}{r}}\right] .
\end{aligned}
$$




\section{Letting}

$$
\begin{aligned}
& L_{1}\left(\mathrm{q}, a_{1}, a_{2}, x\right)=\int_{0}^{\wp_{\theta}(x)} v^{p} d_{\mathrm{q}} v=\left[\wp_{\theta}(x)\right]^{p+1} \frac{1-\mathrm{q}}{1-\mathrm{q}^{p+1}}, \\
& L_{2}\left(\mathrm{q}, a_{1}, a_{2}, x\right)=\int_{0}^{\wp_{\theta}(x)}(1-v)_{0} d_{\mathrm{q}} v=\wp_{\theta}(x)-\frac{1}{1+\mathrm{q}}\left[\wp_{\theta}(x)\right]^{2}, \\
& L_{3}\left(\mathrm{q}, a_{1}, a_{2}, x\right)=\int_{0}^{\wp_{\theta}(x)} v_{0} d_{\mathrm{q}} v=\frac{1}{1+\mathrm{q}}\left[\wp_{\theta}(x)\right]^{2}, \\
& L_{4}\left(\mathrm{q}, a_{1}, a_{2}, x\right)=\int_{\wp_{\theta}(x)}^{1}\left(v-\frac{1}{\mathrm{q}}\right)^{p} d_{\mathrm{q}} v=(1-\mathrm{q})\left[\sum_{n=0}^{\infty} \mathrm{q}^{n}\left(\mathrm{q}^{n}-\frac{1}{\mathrm{q}}\right)^{p}-\wp_{\theta}(x) \sum_{n=0}^{\infty} \mathrm{q}^{n}\left(\mathrm{q}^{n} \wp_{\theta}(x)-\frac{1}{\mathrm{q}}\right)^{p}\right], \\
& L_{5}\left(\mathrm{q}, a_{1}, a_{2}, x\right)=\int_{\wp_{\theta}(x)}^{1}(1-v)_{0} d_{\mathrm{q}} v=\frac{\mathrm{q}}{1+\mathrm{q}}-\wp_{\theta}(x)+\frac{1}{1+\mathrm{q}}\left[\wp_{\theta}(x)\right]^{2}, \\
& L_{6}\left(\mathrm{q}, a_{1}, a_{2}, x\right)=\int_{\wp_{\theta}(x)}^{1} v_{0} d_{\mathrm{q}} v=\frac{1}{1+\mathrm{q}}\left(1-\left[\wp_{\theta}(x)\right]^{2}\right),
\end{aligned}
$$

we have the desired result. The proof is complete.

We point out some special cases of Theorem 4 .

Corollary 1. I. Taking $\mathrm{q} \rightarrow 1^{-}$in Theorem 4 , we have

$$
\begin{aligned}
\left|Y(x)-\frac{1}{\theta\left(a_{2}, a_{1}\right)} \int_{a_{1}}^{a_{1}+\theta\left(a_{2}, a_{1}\right)} \mathrm{Y}(v) d v\right| \\
\leq \theta\left(a_{2}, a_{1}\right)\left[\left[L_{7}\left(a_{1}, a_{2}, x\right)\right]^{\frac{1}{p}}\left[\left|\mathrm{Y}^{\prime}\left(a_{1}\right)\right|^{r} L_{8}\left(a_{1}, a_{2}, x\right)+\left|\mathrm{Y}^{\prime}\left(a_{2}\right)\right|^{r} L_{9}\left(a_{1}, a_{2}, x\right)\right]^{\frac{1}{r}}\right. \\
\left.+\left[L_{10}\left(a_{1}, a_{2}, x\right)\right]^{\frac{1}{p}}\left[\left|\mathrm{Y}^{\prime}\left(a_{1}\right)\right|^{r} L_{11}\left(a_{1}, a_{2}, x\right)+\left|\mathrm{Y}^{\prime}\left(a_{2}\right)\right|^{r} L_{12}\left(a_{1}, a_{2}, x\right)\right]^{\frac{1}{r}}\right],
\end{aligned}
$$


where

$$
\begin{aligned}
& L_{7}\left(a_{1}, a_{2}, x\right)=\int_{0}^{\wp_{\theta}(x)} v^{p} d v=\frac{1}{p+1}\left[\wp_{\theta}(x)\right]^{p+1}, \\
& L_{8}\left(a_{1}, a_{2}, x\right)=\int_{0}^{\wp_{\theta}(x)}(1-v) d v=\wp_{\theta}(x)-\frac{1}{2}\left[\wp_{\theta}(x)\right]^{2}, \\
& L_{9}\left(a_{1}, a_{2}, x\right)=\int_{0}^{\wp_{\theta}(x)} v d v=\frac{1}{2}\left[\wp_{\theta}(x)\right]^{2}, \\
& L_{10}\left(a_{1}, a_{2}, x\right)=\int_{\wp_{\theta}(x)}^{1}(1-v)^{p} d v=\frac{1}{p+1}\left(\frac{a_{1}+\theta\left(a_{2}, a_{1}\right)-x}{\theta\left(a_{2}, a_{1}\right)}\right)^{p+1}, \\
& L_{11}\left(a_{1}, a_{2}, x\right)=\int_{\wp_{\theta}(x)}^{1}(1-v) d v=\frac{a_{1}+\theta\left(a_{2}, a_{1}\right)-x}{\theta\left(a_{2}, a_{1}\right)}-\frac{1}{2}\left(1-\left[\wp_{\theta}(x)\right]^{2}\right), \\
& L_{12}\left(a_{1}, a_{2}, x\right)=\int_{\wp_{\theta}(x)}^{1} v d v=\frac{1}{2}\left(1-\left[\wp_{\theta}(x)\right]^{2}\right) .
\end{aligned}
$$

II. Taking $\mathrm{q} \rightarrow 1^{-}$and $x=\frac{2 a_{1}+\theta\left(a_{2}, a_{1}\right)}{2}$ in Theorem 4 , we get ([13], Theorem 6).

$$
\begin{aligned}
& \left|\mathrm{Y}\left(\frac{2 a_{1}+\theta\left(a_{2}, a_{1}\right)}{2}\right)-\frac{1}{\theta\left(a_{2}, a_{1}\right)} \int_{a_{1}}^{a_{1}+\theta\left(a_{2}, a_{1}\right)} \mathrm{Y}(v) d v\right| \\
& \leq \frac{\theta\left(a_{2}, a_{1}\right)}{16}\left(\frac{4}{p+1}\right)^{\frac{1}{p}}\left[\left(3\left|Y^{\prime}\left(a_{1}\right)\right|^{r}+\left|Y^{\prime}\left(a_{2}\right)\right|^{r}\right)^{\frac{1}{r}}+\left(\left|Y^{\prime}\left(a_{1}\right)\right|^{r}+3\left|Y^{\prime}\left(a_{2}\right)\right|^{r}\right)^{\frac{1}{r}}\right] .
\end{aligned}
$$

III. Taking $x=\frac{q a_{1}+a_{2}}{1+q}$ and $\theta\left(a_{2}, a_{1}\right)=a_{2}-a_{1}$ in Theorem 4, we obtain ([18], Theorem 18).

$$
\begin{aligned}
& \left|\mathrm{Y}\left(\frac{q a_{1}+a_{2}}{1+\mathrm{q}}\right)-\frac{1}{a_{2}-a_{1}} \int_{a_{1}}^{a_{2}} \mathrm{Y}(v)_{a_{1}} d_{\mathrm{q}} v\right| \\
& \leq \mathrm{q}\left(a_{2}-a_{1}\right)\left[\left(\frac{1}{(1+\mathrm{q})^{p+1}} \frac{(1-\mathrm{q})}{1-\mathrm{q}^{p+1}}\right)\left[\frac{\left.\left.\left(\mathrm{q}^{2}+2 \mathrm{q}\right)\right|_{a_{1}} D_{\mathrm{q}} \mathrm{Y}\left(a_{1}\right)\right|^{r}}{(1+\mathrm{q})^{3}}+\frac{\mathrm{q}^{2}\left|a_{1} D_{\mathrm{q}} \mathrm{Y}\left(a_{2}\right)\right|^{r}}{(1+\mathrm{q})^{3}}\right]^{\frac{1}{r}}\right. \\
& \left.+\left(\int_{\frac{1}{1+\mathrm{q}}}^{1}\left(v-\frac{1}{\mathrm{q}}\right)^{p} a_{1} d_{\mathrm{q}} v\right)^{\frac{1}{p}}\left[\frac{\left(\mathrm{q}^{3}+\mathrm{q}^{2}-\mathrm{q}\right)\left|a_{1} D_{\mathrm{q}} \mathrm{Y}\left(a_{1}\right)\right|^{r}}{(1+\mathrm{q})^{3}}+\frac{\left(\mathrm{q}^{2}+2 \mathrm{q}\right)\left|a_{1} D_{\mathrm{q}} \mathrm{Y}\left(a_{2}\right)\right|^{r}}{(1+\mathrm{q})^{3}}\right]^{\frac{1}{r}}\right],
\end{aligned}
$$

where

$$
\int_{\frac{1}{1+\mathrm{q}}}^{1}\left(v-\frac{1}{\mathrm{q}}\right)^{p}{ }_{0} d_{\mathrm{q}} v=(1-\mathrm{q})\left[\sum_{n=0}^{\infty} \mathrm{q}^{n}\left(\mathrm{q}^{n}-\frac{1}{\mathrm{q}}\right)^{p}-\frac{1}{1+\mathrm{q}} \sum_{n=0}^{\infty} \mathrm{q}^{n}\left(\mathrm{q}^{n}\left(\frac{1}{1+\mathrm{q}}\right)-\frac{1}{\mathrm{q}}\right)^{p}\right] .
$$


IV. Taking $x=\frac{a_{1}+\mathrm{q}\left(a_{1}+\theta\left(a_{2}, a_{1}\right)\right)}{1+\mathrm{q}}$ in Theorem 4 , we get

$$
\begin{aligned}
& \left|\mathrm{Y}\left(\frac{a_{1}+\mathrm{q}\left(a_{1}+\theta\left(a_{2}, a_{1}\right)\right)}{1+\mathrm{q}}\right)-\frac{1}{\theta\left(a_{2}, a_{1}\right)} \int_{a_{1}}^{a_{1}+\theta\left(a_{2}, a_{1}\right)} \mathrm{Y}(v) a_{1} d_{\mathrm{q}} v\right| \\
& \leq \mathrm{q} \theta\left(a_{2}, a_{1}\right)\left[\left(\frac{\mathrm{q}^{p}}{(1+\mathrm{q})^{p+1}} \frac{(1-\mathrm{q})}{1-\mathrm{q}^{p+1}}\right)\left[\frac{\left.\left.\left(\mathrm{q}^{3}+\mathrm{q}^{2}+\mathrm{q}\right)\right|_{a_{1}} D_{\mathrm{q}} \mathrm{Y}\left(a_{1}\right)\right|^{r}}{(1+\mathrm{q})^{3}}+\frac{\left.\left.\mathrm{q}^{2}\right|_{a_{1}} D_{\mathrm{q}} \mathrm{Y}\left(a_{2}\right)\right|^{r}}{(1+\mathrm{q})^{3}}\right]^{\frac{1}{r}}\right. \\
& \left.+\left(\int_{\frac{\mathrm{q}}{1+\mathrm{q}}}^{1}\left(v-\frac{1}{\mathrm{q}}\right)^{p}{ }_{0} d_{\mathrm{q}} v\right)^{\frac{1}{p}}\left[\frac{\mathrm{q}^{2}\left|a_{1} D_{\mathrm{q}} \mathrm{Y}\left(a_{1}\right)\right|^{r}}{(1+\mathrm{q})^{3}}+\frac{\left.\left.(1+2 \mathrm{q})\right|_{a_{1}} D_{\mathrm{q}} \mathrm{Y}\left(a_{2}\right)\right|^{r}}{(1+\mathrm{q})^{3}}\right]^{\frac{1}{r}}\right],
\end{aligned}
$$

where

$$
\int_{\frac{\mathrm{q}}{1+\mathrm{q}}}^{1}\left(v-\frac{1}{\mathrm{q}}\right)^{p}{ }_{0} d_{\mathrm{q}} v=(1-\mathrm{q})\left[\sum_{n=0}^{\infty} \mathrm{q}^{n}\left(\mathrm{q}^{n}-\frac{1}{\mathrm{q}}\right)^{p}-\frac{\mathrm{q}}{1+\mathrm{q}} \sum_{n=0}^{\infty} \mathrm{q}^{n}\left(\mathrm{q}^{n}\left(\frac{\mathrm{q}}{1+\mathrm{q}}\right)-\frac{1}{\mathrm{q}}\right)^{p}\right]
$$

Theorem 5. Let $\mathrm{Y}: P \rightarrow \mathbb{R}$ be a function such that $a_{1} D_{\mathrm{q}} \mathrm{Y}$ is q-integrable on $P^{\circ}$ (the interior of $P$ ). If $\left|a_{1} D_{\mathrm{q}} \mathrm{Y}\right|^{r}$ is preinvex function on $P$, then for $r \geq 1$, the following inequality holds:

$$
\begin{aligned}
& \left|\mathrm{Y}(x)-\frac{1}{\theta\left(a_{2}, a_{1}\right)} \int_{a_{1}}^{a_{1}+\theta\left(a_{2}, a_{1}\right)} \mathrm{Y}(v) a_{1} d_{\mathrm{q}} v\right| \leq \theta\left(a_{2}, a_{1}\right) \times \\
& \quad\left[\left[J_{1}\left(\mathrm{q}, a_{1}, a_{2}, x\right)\right]^{1-\frac{1}{r}}\left[\left|a_{1} D_{\mathrm{q}} \mathrm{Y}\left(a_{1}\right)\right|^{r} J_{2}\left(\mathrm{q}, a_{1}, a_{2}, x\right)+\left|a_{1} D_{\mathrm{q}} \mathrm{Y}\left(a_{2}\right)\right|^{r} J_{3}\left(\mathrm{q}, a_{1}, a_{2}, x\right)\right]^{\frac{1}{r}}\right. \\
& \left.+\left[J_{4}\left(\mathrm{q}, a_{1}, a_{2}, x\right)\right]^{1-\frac{1}{r}}\left[\left|a_{1} D_{\mathrm{q}} \mathrm{Y}\left(a_{1}\right)\right|^{r} J_{5}\left(\mathrm{q}, a_{1}, a_{2}, x\right)+\left|a_{1} D_{\mathrm{q}} \mathrm{Y}\left(a_{2}\right)\right|^{r} J_{6}\left(\mathrm{q}, a_{1}, a_{2}, x\right)\right]^{\frac{1}{r}}\right],
\end{aligned}
$$

where

$$
\begin{aligned}
& J_{1}\left(\mathrm{q}, a_{1}, a_{2}, x\right)=\int_{0}^{\wp_{\theta}(x)} \mathrm{q} v_{0} d_{\mathrm{q}} v=\frac{\mathrm{q}}{1+\mathrm{q}}\left[\wp_{\theta}(x)\right]^{2}, \\
& J_{2}\left(\mathrm{q}, a_{1}, a_{2}, x\right)=\int_{0}^{\wp_{\theta}(x)}\left(\mathrm{q} v-\mathrm{q} v^{2}\right)_{0} d_{\mathrm{q}} v=J_{1}\left(\mathrm{q}, a_{1}, a_{2}, x\right)-J_{3}\left(\mathrm{q}, a_{1}, a_{2}, x\right), \\
& J_{3}\left(\mathrm{q}, a_{1}, a_{2}, x\right)=\int_{0}^{\wp_{\theta}(x)} \mathrm{q}^{2}{ }_{0} d_{\mathrm{q}} v=\frac{\mathrm{q}}{1+\mathrm{q}+\mathrm{q}^{2}}\left[\wp_{\theta}(x)\right]^{3}, \\
& J_{4}\left(\mathrm{q}, a_{1}, a_{2}, x\right)=\int_{\wp_{\theta}(x)}^{1}(\mathrm{q} v-1){ }_{0} d_{\mathrm{q}} v=\frac{\mathrm{q}}{1+\mathrm{q}}\left(\frac{a_{1}+\theta\left(a_{2}, a_{1}\right)-x}{\theta\left(a_{2}, a_{1}\right)}\right)^{2}, \\
& J_{5}\left(\mathrm{q}, a_{1}, a_{2}, x\right)=\int_{\wp_{\theta}(x)}^{1}\left(1-\mathrm{q} v-v+\mathrm{q} v^{2}\right)_{0} d_{\mathrm{q}} v=J_{4}\left(\mathrm{q}, a_{1}, a_{2}, x\right)-J_{6}\left(\mathrm{q}, a_{1}, a_{2}, x\right), \\
& J_{6}\left(\mathrm{q}, a_{1}, a_{2}, x\right)=\int_{\wp_{\theta}(x)}^{1}\left(\mathrm{q} v^{2}-v\right)_{0} d_{\mathrm{q}} v=\frac{1}{(1+\mathrm{q})\left(1+\mathrm{q}+\mathrm{q}^{2}\right)}-\frac{1}{1+\mathrm{q}}\left[\wp_{\theta}(x)\right]^{2}+\frac{\mathrm{q}}{1+\mathrm{q}+\mathrm{q}^{2}}\left[\wp_{\theta}(x)\right]^{3} .
\end{aligned}
$$


Proof. Using Lemma 2, preinvexity of $\left|a_{1} D_{\mathrm{q}} \mathrm{Y}\right|^{r}$ and the well-known power mean inequality, we have

$$
\begin{aligned}
& \left|\mathrm{Y}(x)-\frac{1}{\theta\left(a_{2}, a_{1}\right)} \int_{a_{1}}^{a_{1}+\theta\left(a_{2}, a_{1}\right)} \mathrm{Y}(v)_{a_{1}} d_{\mathrm{q}} v\right| \\
& \leq \theta\left(a_{2}, a_{1}\right)\left[\left.\left.\int_{0}^{\wp_{\theta}(x)} \mathrm{q} v\right|_{a_{1}} D_{\mathrm{q}} \mathrm{Y}\left(a_{1}+v \theta\left(a_{2}, a_{1}\right)\right)\right|_{0} d_{\mathrm{q}} v+\left.\left.\int_{\gamma_{\theta}(x)}^{1}(\mathrm{q} v-1)\right|_{a_{1}} D_{\mathrm{q}} \mathrm{Y}\left(a_{1}+v \theta\left(a_{2}, a_{1}\right)\right)\right|_{0} d_{\mathrm{q}} v\right] \\
& \leq \theta\left(a_{2}, a_{1}\right)\left[\left(\int_{0}^{\wp_{\theta}(x)} \mathrm{q} v_{0} d_{\mathrm{q}} v\right)^{1-\frac{1}{r}}\left(\left.\left.\int_{0}^{\wp_{\theta}(x)} \mathrm{q} v\right|_{a_{1}} D_{\mathrm{q}} \mathrm{Y}\left(a_{1}+v \theta\left(a_{2}, a_{1}\right)\right)\right|^{r} 0 d_{\mathrm{q}} v\right)^{\frac{1}{r}}\right. \\
& \left.+\left(\int_{\rho_{\theta}(x)}^{1}(\mathrm{q} v-1)_{0} d_{\mathrm{q}} v\right)^{1-\frac{1}{r}}\left(\left.\left.\int_{\rho_{\theta}(x)}^{1}(\mathrm{q} v-1)\right|_{a_{1}} D_{\mathrm{q}} \mathrm{Y}\left(a_{1}+v \theta\left(a_{2}, a_{1}\right)\right)\right|^{r}{ }_{0} d_{\mathrm{q}} v\right)^{\frac{1}{r}}\right] \\
& \leq \theta\left(a_{2}, a_{1}\right)\left[\left(\int_{0}^{\wp_{\theta}(x)} \mathrm{q} v d_{0} v\right)^{1-\frac{1}{r}}\left(\left|a_{1} D_{\mathrm{q}} \mathrm{Y}\left(a_{1}\right)\right|^{r} \int_{0}^{\wp_{\theta}(x)}\left(\mathrm{q} v-\mathrm{q} v^{2}\right)_{0} d_{\mathrm{q}} v+\left|a_{1} D_{\mathrm{q}} \mathrm{Y}\left(a_{2}\right)\right|^{r} \int_{0}^{\wp_{\theta}(x)} \mathrm{q} v^{2}{ }_{0} d_{\mathrm{q}} v\right)^{\frac{1}{r}}\right. \\
& \left.+\left(\int_{\rho_{\theta}(x)}^{1}(\mathrm{q} v-1)_{0} d_{\mathrm{q}} v\right)^{1-\frac{1}{r}}\left(\left|a_{a_{1}} D_{\mathrm{q}} \mathrm{Y}\left(a_{1}\right)\right|^{r} \int_{\rho_{\theta}(x)}^{1}\left(1-\mathrm{q} v-v+\mathrm{q} v^{2}\right)_{0} d_{\mathrm{q}} v+\left.\left.\right|_{a_{1}} D_{\mathrm{q}} \mathrm{Y}\left(a_{2}\right)\right|^{r} \int_{\rho_{\theta}(x)}^{1}\left(\mathrm{q} v^{2}-v\right)_{0} d_{\mathrm{q}} v\right)^{\frac{1}{r}}\right] .
\end{aligned}
$$

The proof of Theorem 5 is completed.

We point out some special cases of Theorem 5 .

Corollary 2. I. Taking $r=1$ in Theorem 5, we have

$$
\begin{array}{r}
\left|\mathrm{Y}(x)-\frac{1}{\theta\left(a_{2}, a_{1}\right)} \int_{a_{1}}^{a_{1}+\theta\left(a_{2}, a_{1}\right)} \mathrm{Y}(v)_{a_{1}} d_{\mathrm{q}} v\right| \leq \theta\left(a_{2}, a_{1}\right)\left[{ }_{a_{1}} D_{\mathrm{q}} \mathrm{Y}\left(a_{1}\right) \mid\left[J_{2}\left(\mathrm{q}, a_{1}, a_{2}, x\right)+J_{5}\left(\mathrm{q}, a_{1}, a_{2}, x\right)\right]\right. \\
\left.+\left.\right|_{a_{1}} D_{\mathrm{q}} \mathrm{Y}\left(a_{2}\right) \mid\left[J_{3}\left(\mathrm{q}, a_{1}, a_{2}, x\right)+J_{6}\left(\mathrm{q}, a_{1}, a_{2}, x\right)\right]\right] .
\end{array}
$$

II. Taking $r=1$ and $\mathrm{q} \rightarrow 1^{-}$in Theorem 5 , we get

$$
\begin{array}{r}
\left|Y(x)-\frac{1}{\theta\left(a_{2}, a_{1}\right)} \int_{a_{1}}^{a_{1}+\theta\left(a_{2}, a_{1}\right)} Y(v) d v\right| \leq \theta\left(a_{2}, a_{1}\right)\left[\left|Y^{\prime}\left(a_{1}\right)\right|\left[J_{7}\left(a_{1}, a_{2}, x\right)+J_{9}\left(a_{1}, a_{2}, x\right)\right]\right. \\
\left.+\left|Y^{\prime}\left(a_{2}\right)\right|\left[J_{8}\left(a_{1}, a_{2}, x\right)+J_{10}\left(a_{1}, a_{2}, x\right)\right]\right]
\end{array}
$$


where

$$
\begin{aligned}
& J_{7}\left(a_{1}, a_{2}, x\right)=\int_{0}^{\wp_{\theta}(x)} v(1-v) d v=\frac{1}{2}\left[\wp_{\theta}(x)\right]^{2}-\frac{1}{3}\left[\wp_{\theta}(x)\right]^{3}, \\
& J_{8}\left(a_{1}, a_{2}, x\right)=\int_{0}^{\wp_{\theta}(x)} v^{2} d v=\frac{1}{3}\left[\wp_{\theta}(x)\right]^{3}, \\
& J_{9}\left(a_{1}, a_{2}, x\right)=\int_{\wp_{\theta}(x)}^{1}\left(1-2 v+v^{2}\right) d v=\frac{1}{3}-\wp_{\theta}(x)+\left[\wp_{\theta}(x)\right]^{2}-\frac{1}{3}\left[\wp_{\theta}(x)\right]^{3}, \\
& J_{10}\left(a_{1}, a_{2}, x\right)=\int_{\wp_{\theta}(x)}^{1}\left(v-v^{2}\right) d v=\frac{1}{6}-\frac{1}{2}\left[\wp_{\theta}(x)\right]^{2}+\frac{1}{3}\left[\wp_{\theta}(x)\right]^{3} .
\end{aligned}
$$

III. Taking $\mathrm{q} \rightarrow 1^{-}$and $x=\frac{2 a_{1}+\theta\left(a_{2}, a_{1}\right)}{2}$ in Theorem 5, we obtain ([13], Theorem 8).

$$
\begin{aligned}
& \left|\mathrm{Y}\left(\frac{2 a_{1}+\theta\left(a_{2}, a_{1}\right)}{\theta\left(a_{2}, a_{1}\right)}\right)-\frac{1}{\theta\left(a_{2}, a_{1}\right)} \int_{a_{1}}^{a_{1}+\theta\left(a_{2}, a_{1}\right)} \mathrm{Y}(v) d v\right| \\
& \leq \frac{\theta\left(a_{2}, a_{1}\right)}{8}\left[\left(\frac{2\left|\mathrm{Y}^{\prime}\left(a_{1}\right)\right|^{r}+\left|\mathrm{Y}^{\prime}\left(a_{2}\right)\right|^{r}}{3}\right)^{\frac{1}{r}}+\left(\frac{\left|\mathrm{Y}^{\prime}\left(a_{1}\right)\right|^{r}+2\left|\mathrm{Y}^{\prime}\left(a_{2}\right)\right|^{r}}{3}\right)^{\frac{1}{r}}\right] .
\end{aligned}
$$

IV. Taking $r=1, \mathrm{q} \rightarrow 1^{-}$and $x=\frac{2 a_{1}+\theta\left(a_{2}, a_{1}\right)}{2}$ in Theorem 5, we get ([13], Theorem 5).

$$
\left|Y\left(\frac{2 a_{1}+\theta\left(a_{2}, a_{1}\right)}{2}\right)-\frac{1}{\theta\left(a_{2}, a_{1}\right)} \int_{a_{1}}^{a_{1}+\theta\left(a_{2}, a_{1}\right)} Y(v) d v\right| \leq \frac{\theta\left(a_{2}, a_{1}\right)}{8}\left[\left|Y^{\prime}\left(a_{1}\right)\right|+\left|Y^{\prime}\left(a_{2}\right)\right|\right] .
$$

IV. Taking $x=\frac{q a_{1}+a_{2}}{1+q}$ and $\theta\left(a_{2}, a_{1}\right)=a_{2}-a_{1}$ in Theorem 5 , we have the following inequalities, for more details, see [20].

$$
\begin{aligned}
& \left|\mathrm{Y}\left(\frac{q a_{1}+a_{2}}{1+\mathrm{q}}\right)-\frac{1}{a_{2}-a_{1}} \int_{a_{1}}^{a_{2}} \mathrm{Y}(v) a_{1} d_{\mathrm{q}} v\right| \\
& \leq\left(a_{2}-a_{1}\right)\left[\frac{1}{(1+\mathrm{q})^{3-\frac{3}{r}}}\left[\left|a_{1} D_{\mathrm{q}} \mathrm{Y}\left(a_{1}\right)\right|^{r} \frac{\mathrm{q}^{2}(1+\mathrm{q})}{\left(1+\mathrm{q}+\mathrm{q}^{2}\right)(1+\mathrm{q})^{3}}+\left|a_{a_{1}} D_{\mathrm{q}} \mathrm{Y}\left(a_{2}\right)\right|^{r} \frac{\mathrm{q}}{\left(1+\mathrm{q}+\mathrm{q}^{2}\right)(1+\mathrm{q})^{3}}\right]^{\frac{1}{r}}\right. \\
& \left.+\left(\frac{\mathrm{q}}{1+\mathrm{q}}\right)^{3-\frac{3}{r}}\left[\left|a_{1} D_{\mathrm{q}} \mathrm{Y}\left(a_{1}\right)\right|^{r} \frac{\left(\mathrm{q}^{5}+\mathrm{q}^{4}+\mathrm{q}^{3}-2 \mathrm{q}\right)}{\left(1+\mathrm{q}+\mathrm{q}^{2}\right)(1+\mathrm{q})^{3}}+\left|a_{1} D_{\mathrm{q}} \mathrm{Y}\left(a_{2}\right)\right|^{r} \frac{2 \mathrm{q}}{\left(1+\mathrm{q}+\mathrm{q}^{2}\right)(1+\mathrm{q})^{3}}\right]^{\frac{1}{r}}\right] .
\end{aligned}
$$

V. Taking $r=1, x=\frac{q a_{1}+a_{2}}{1+\mathrm{q}}$ and $\theta\left(a_{2}, a_{1}\right)=a_{2}-a_{1}$ in Theorem 5, we obtain ([18], Theorem 13).

$$
\begin{aligned}
& \left|\mathrm{Y}\left(\frac{q a_{1}+a_{2}}{1+\mathrm{q}}\right)-\frac{1}{a_{2}-a_{1}} \int_{a_{1}}^{a_{2}} \mathrm{Y}(v) a_{1} d_{\mathrm{q}} v\right| \\
& \leq\left(a_{2}-a_{1}\right)\left[\left|a_{1} D_{\mathrm{q}} \mathrm{Y}\left(a_{1}\right)\right| \frac{\left(\mathrm{q}^{5}+\mathrm{q}^{4}+2 \mathrm{q}^{3}+\mathrm{q}^{2}-2 \mathrm{q}\right)}{\left(1+\mathrm{q}+\mathrm{q}^{2}\right)(1+\mathrm{q})^{3}}+\left.\right|_{a_{1}} D_{\mathrm{q}} \mathrm{Y}\left(a_{2}\right) \mid \frac{3 \mathrm{q}}{\left(1+\mathrm{q}+\mathrm{q}^{2}\right)(1+\mathrm{q})^{3}}\right] .
\end{aligned}
$$


VI. Taking $x=\frac{a_{1}+\mathrm{q}\left(a_{1}+\theta\left(a_{2}, a_{1}\right)\right)}{1+\mathrm{q}}$ in Theorem 5 , we get

$$
\begin{aligned}
& \left|\mathrm{Y}\left(\frac{a_{1}+\mathrm{q}\left(a_{1}+\theta\left(a_{2}, a_{1}\right)\right)}{1+\mathrm{q}}\right)-\frac{1}{\theta\left(a_{2}, a_{1}\right)} \int_{a_{1}}^{a_{1}+\theta\left(a_{2}, a_{1}\right)} \mathrm{Y}(v)_{a_{1}} a_{\mathrm{q}} v\right| \leq \theta\left(a_{2}, a_{1}\right) \times \\
& {\left[\left(\frac{\mathrm{q}}{1+\mathrm{q}}\right)^{3-\frac{3}{r}}\left[\left|a_{1} D_{\mathrm{q}} \mathrm{Y}\left(a_{1}\right)\right|^{r} \frac{\mathrm{q}^{3}\left(1+\mathrm{q}^{2}\right)}{\left(1+\mathrm{q}+\mathrm{q}^{2}\right)(1+\mathrm{q})^{3}}+\left|a_{1} D_{\mathrm{q}} \mathrm{Y}\left(a_{2}\right)\right|^{r} \frac{\mathrm{q}^{4}}{\left(1+\mathrm{q}+\mathrm{q}^{2}\right)(1+\mathrm{q})^{3}}\right]^{\frac{1}{r}}\right.} \\
& \left.+\left(\frac{\mathrm{q}}{(1+\mathrm{q})^{3}}\right)^{1-\frac{1}{r}}\left[\left|a_{1} D_{\mathrm{q}} \mathrm{Y}\left(a_{1}\right)\right|^{r} \frac{\left(2 \mathrm{q}^{3}+\mathrm{q}^{2}-\mathrm{q}-1\right)}{\left(1+\mathrm{q}+\mathrm{q}^{2}\right)(1+\mathrm{q})^{3}}+\left|a_{1} D_{\mathrm{q}} \mathrm{Y}\left(a_{2}\right)\right|^{r} \frac{\left(1+2 \mathrm{q}-\mathrm{q}^{3}\right)}{\left(1+\mathrm{q}+\mathrm{q}^{2}\right)(1+\mathrm{q})^{3}}\right]^{\frac{1}{r}}\right] .
\end{aligned}
$$

\section{Applications to Special Means}

Recalling the following means for arbitrary real numbers $a$ and $b$ with $a \neq b$ :

$$
\begin{array}{ll}
A\left(a_{1}, a_{2}\right)=\frac{a_{1}+a_{2}}{2} & \text { Arithmetic mean } \\
L_{p}\left(a_{1}, a_{2}\right)=\left(\frac{a_{2}^{p+1}-a_{1}^{p+1}}{(p+1)\left(a_{2}-a_{1}\right)}\right)^{1 / p}, p \in \mathbb{R} \backslash\{-1,0\} & \text { p- Logarithmic mean }
\end{array}
$$

it is possible to relate them through the previous results.

Proposition 1. Let $p \in \mathbb{R} \backslash\{-1,0\}$ and $a_{1}, a_{2}$ real numbers such that $a_{2} \geq a_{2}$, then

$$
\left|A^{p}\left(a_{1}, a_{2}\right)-\frac{1}{a_{2}-a_{1}} L_{p}^{p}\left(a_{1}, a_{2}\right)\right| \leq \frac{|p|}{8}\left(a_{2}-a_{1}\right)\left(\left|a_{1}^{p-1}\right|+\left|a_{2}^{p-1}\right|\right)
$$

Proof. Let $f(x)=x^{p}$ for some arbitrary $p \in \mathbb{R} \backslash\{-1,0\}, x=\left(q a_{1}+a_{2}\right) /(1+q)$ and $r=1$. Then

$$
\begin{gathered}
f\left(\frac{q a_{1}+a_{2}}{1+q}\right)=\left(\frac{q a_{1}+a_{2}}{1+q}\right)^{p}, \\
\int_{a_{1}}^{a_{2}} t^{p}{ }_{a_{1}} d_{q} t=\left[\frac{1-q}{1-q^{p+1}}\right]\left(\frac{a_{2}^{p+1}-a_{1}^{p+1}}{a_{2}-a_{1}}\right)
\end{gathered}
$$

and

$$
a_{1} D_{\mathrm{q}} \mathrm{Y}\left(a_{1}\right)=\left[\frac{1-q^{p}}{1-q}\right] a_{1}^{p-1} \text { and } a_{1} D_{\mathrm{q}} \mathrm{Y}\left(a_{2}\right)=\left[\frac{1-q^{p}}{1-q}\right] a_{2}^{p-1}
$$

So, using the Corollary 2 part IV, we have

$$
\begin{aligned}
& \left|\left(\frac{q a_{1}+a_{2}}{1+q}\right)^{p}-\frac{1}{a_{2}-a_{1}}\left[\frac{1-q}{1-q^{p+1}}\right]\left(\frac{a_{2}^{p+1}-a_{1}^{p+1}}{a_{2}-a_{1}}\right)\right| \\
& \leq\left(a_{2}-a_{1}\right)\left[\frac{1}{(1+\mathrm{q})^{3-\frac{3}{r}}}\left[\left|\left[\frac{1-q^{p}}{1-q}\right] a_{1}^{p-1}\right| \frac{\mathrm{q}^{2}(1+\mathrm{q})}{\left(1+\mathrm{q}+\mathrm{q}^{2}\right)(1+\mathrm{q})^{3}}+\left|\left[\frac{1-q^{p}}{1-q}\right] a_{2}^{p-1}\right| \frac{\mathrm{q}}{\left(1+\mathrm{q}+\mathrm{q}^{2}\right)(1+\mathrm{q})^{3}}\right]^{\frac{1}{r}}\right. \\
& \left.+\left(\frac{\mathrm{q}}{1+\mathrm{q}}\right)^{3-\frac{3}{r}}\left[\left|\left[\frac{1-q^{p}}{1-q}\right] a_{1}^{p-1}\right| \frac{\left(\mathrm{q}^{5}+\mathrm{q}^{4}+\mathrm{q}^{3}-2 \mathrm{q}\right)}{\left(1+\mathrm{q}+\mathrm{q}^{2}\right)(1+\mathrm{q})^{3}}+\left[\mid \frac{1-q^{p}}{1-q}\right] a_{2}^{p-1} \mid \frac{2 \mathrm{q}}{\left(1+\mathrm{q}+\mathrm{q}^{2}\right)(1+\mathrm{q})^{3}}\right]^{\frac{1}{r}}\right] .
\end{aligned}
$$


Taking limit when $q \rightarrow 1$

$$
\begin{aligned}
& \left|\left(\frac{a_{1}+a_{2}}{2}\right)^{p}-\frac{1}{a_{2}-a_{1}}\left(\frac{a_{2}^{p+1}-a_{1}^{p+1}}{(p+1)\left(a_{2}-a_{1}\right)}\right)\right| \\
& \leq\left(a_{2}-a_{1}\right)\left[\left|p a_{1}^{p-1}\right| \frac{1}{12}+\left|p a_{2}^{p-1}\right| \frac{1}{24}+\left|p a_{1}^{p-1}\right| \frac{1}{24}+\left|p a_{2}^{p-1}\right| \frac{1}{12}\right] \\
& \leq \frac{|p|}{8}\left(a_{2}-a_{1}\right)\left(\left|a_{1}^{p-1}\right|+\left|a_{2}^{p-1}\right|\right) .
\end{aligned}
$$

The proof is complete.

\section{Conclusions}

It is expected that from the results obtained, and following the methodology applied, additional special functions may also be evaluated. Future works can be developed in the area of numerical analysis and even contributions using quantum algorithms, using the theorems and corollaries presented. Finally, our results can be applied to derive some inequalities using special means. The authors hope that the ideas and techniques of this paper will inspire interested readers working in this fascinating field.

Author Contributions: All authors contributed equally in the preparation of the present work taking into account the theorems and corollaries presented, the review of the articles and books cited, formal analysis, investigation, writing-original draft preparation and writing-review and editing. All authors have read and agreed to the published version of the manuscript.

Funding: This research was funded by Dirección de Investigación from Pontificia Universidad Católica del Ecuador in the research project entitled: Some inequalities using generalized convexity. All authors have read and agreed to the published version of the manuscript.

Acknowledgments: Miguel J. Vivas-Cortez thanks to Dirección de Investigación from Pontificia Universidad Católica del Ecuador for the technical support given to the research project entitled: Algunas desigualdades de funciones convexas generalizadas (Some inequalities of generalized convex functions). Jorge E. Hernández Hernández wants to thank to the Consejo de Desarrollo Científico, Humanístico y Tecnológico (CDCHT) from Universidad Centroccidental Lisandro Alvarado (Venezuela), also for the technical support given in the development of this article.

Conflicts of Interest: The authors declare no conflict of interest.

\section{References}

1. Ernst T. $q$-Calculus and physics. In A Comprehensive Treatment of $q$-Calculus; Birkhäuser: Basel, Switzerland, 2012; pp. 441-446

2. Niculescu, C.P. An invitation to convex function theory. In Order Structures in Functional Analysis; Editura Academiei Romane: Bucarest, Romania, 2001.

3. Bennett, C.; Sharpley, R. Interpolation of Operators; Academic Press: Boston, MA, USA, 1998.

4. Mititelu, Ş.; Trenţă, S. Efficiency conditions in vector control problems governed by multiple integrals. J. Appl. Math. Comp. 2018, 57, 647-665. [CrossRef]

5. Trență, S. On a New Class of Vector Variational Control Problems. Numer. Funct. Anal. Optimi. 2018, 39, 1594-1603. [CrossRef]

6. Trenţă, S. KT-geodesic pseudoinvex control problems governed by multiple integrals. J. Nonlin. Conv. Anal. 2019, 20, 73-84.

7. Ben-Israel, A.; Mond, B. What is invexity? J. Austral. Math. Soc. Ser. B 1986, 28, 1-9. [CrossRef]

8. Hernández Hernández, J.E. On Some New Integral Inequalities Related with the Hermite-Hadamard Inequality via h-Convex Functions. MAYFEB J. Math. 2017, 4, 1-12

9. Hernández Hernández, J.E. On log- $\left(m, h_{1}, h_{2}\right)$-convex functionsand related integral inequalities. Int. J. Open Prob. Compt. Math. 2019, 12, 43-59.

10. Mitrinović, D.S.; Pečarić, J.; Fink, A.M. Inequalities for Functions and Their Integrals and Derivatives; Kluwer Academic: Dordrecht, The Netherlands, 1991. 
11. Noor, M.A.; Noor, K.I.; Awan, M.U.; Li, J. On Hermite-Hadamard Inequalities for $h$-preinvex functions. Filomat 2014, 28, 1463-1474. [CrossRef]

12. Noor, M.A.; Cristescu, G.; Awan, M.U. Bounds having Riemann type quantum integrals via strongly convex functions. Studia Sci. Math. Hung. 2017, 54, 221-240. [CrossRef]

13. Sarikaya, M.Z.; Bozkurt, H.; Alp, N. On Hermite-Hadamard type integral inequalities for preinvex and log-preinvex functions. Contemp. Anal. Appl. Math. 2013, 1, 237-252.

14. Vivas-Cortez, M.J.; García, C.; Hernández Hernández, J.E. Ostrowski-Type Inequalities for Functions Whose Derivative Modulus is Relatively $\left(m, h_{1}, h_{2}\right)$-Convex. Appl. Math. Inf. Sci. 2019, 13, 369-378. [CrossRef]

15. Weir, T.; Mond, B. Preinvex functions in multiple objective optimization. J. Math. Anal. Appl. 1988, 136, $29-38$. [CrossRef]

16. Tariboon, J.; Ntouyas, S.K. Quantum integral inequalities on finite intervals. J. Inequal. Appl. 2014, $2014,121$. [CrossRef]

17. Tariboon, J.; Ntouyas, S.K. Quantum calculus on finite intervals and applications to impulsive difference equations. Adv. Differ. Equ. 2013, 2013, 1-19. [CrossRef]

18. Alp, N.; Sarikaya, M.Z.; Kunt, M.; İşcan, İ. q-Hermite Hadamard inequalities and quantum estimates for midpoint type inequalities via convex and quasi-convex functions. J. King Saud-Univ. -Sci. 2018, 30, 193-203. [CrossRef]

19. Kac, V.; Cheung, P. Quantum calculus, Universitext; Springer: New York, NY, USA, 2002.

20. Kunt, M.; Kashuri, A.; Du, T. Quantum Montgomery identity and some quantum integral inequalities. arXiv 2019, arXiv:1907.03601.

21. Mohsin, B.B.; Awan, M.U.; Noor, M.A.; Riahi, L.; Noor, K.I.; Almutairi, B. New quantum Hermite-Hadamard inequalities utilizing harmonic convexity of the functions. IEEE Access 2019, 7, 20479-20483. [CrossRef]

22. Noor, M.A.; Noor, K.I.; Awan, M.U. Some quantum integral inequalities via preinvex functions. Appl. Math. Comput 2015, 269, 242-251. [CrossRef]

23. Noor, M.A.; Noor, K.I.; Awan, M.U. Some quantum estimates for Hermite-Hadamard inequalities. Appl. Math. Comput. 2015, 251, 675-679. [CrossRef]

24. Sudsutad, W.; Ntouyas, S.K.; Tariboon, J. Quantum integral inequalities for convex functions. J. Math. Inequal. 2013, 9 , 781-793. [CrossRef]

25. Vivas-Cortez, M.J.; Liko, R.; Kashuri, A.; Hernández Hernández, J.E, New quantum estimates of trapezium-type inequalities for generalized $\phi$-convex functions. Mathematics 2019, $7,1-19$. [CrossRef]

26. Vivas-Cortez, M.J.; Kashuri, A.; Liko, R.; Hernández Hernández, J.E. Quantum estimates of Ostrowski inequalities for generalized $\phi$-convex functions. Symmetry 2019, 11, 1-16.

27. Vivas-Cortez, M.J.; Kashuri, A.; Liko, R.; Hernández Hernández, J.E. Some inequalities using generalized convex functions in quantum analysis. Symmetry 2019, 11, 1-14. [CrossRef]

28. Vivas-Cortez, M.J.; Kashuri, A.; Liko, R.; Hernández, J.E. Quantum Trapezium-Type Inequalities Using Generalized $\phi$-Convex Functions. Axioms 2020, 9, 1-14 [CrossRef]

29. Zhang, Y.; Du, T.S.; Wang, H.; Shen, Y.J. Different types of quantum integral inequalities via $(\alpha, m)$-convexity. J. Inequal. Appl. 2018, 2018, 1-20. [CrossRef]

30. Ujević, N. An application of the Montgomery identity to quadrature rukles. Rend. Sem. Mat. Univ. Pol. Torino 2008, 66, 137-143

31. Yang, X.M.; Li, D. On Properties of Preinvex Functions. J. Math. Anal. Appl. 2001, 256, 229-241. [CrossRef]

(c) 2020 by the authors. Licensee MDPI, Basel, Switzerland. This article is an open access article distributed under the terms and conditions of the Creative Commons Attribution (CC BY) license (http:/ / creativecommons.org/licenses/by/4.0/). 